\title{
An innovative technique for detecting the caudal end of occluded inferior petrosal sinus in cavernous arteriovenous fistula using intravascular ultrasonography_technical note
}

\author{
Shigeru Yamauchi ${ }^{1} \cdot$ Akimasa Nishio $^{1} \cdot$ Yoshinobu Takahashi $^{1} \cdot$ Kimito Kondo $^{2}$. \\ Taichiro Kawakami $^{3}$ - Yuzo Terakawa ${ }^{3}$ - Yutaka Mitsuhashi ${ }^{4}$ - Kenji Ohata ${ }^{3}$
}

Received: 10 February 2015 / Accepted: 5 April 2015 /Published online: 24 April 2015

(C) The Author(s) 2015. This article is published with open access at Springerlink.com

\begin{abstract}
Introduction Although cavernous sinus (CS) dural arteriovenous fistulas (d-AVFs) are usually treated with transvenous embolization (TVE) via the inferior petrosal sinus (IPS), IPSs are sometimes thrombosed and angiographically invisible. In such cases, the first obstacle to TVE is detecting the entry to the IPS. We report a new technique for TVE via IPS using intravascular ultrasonography (IVUS).

Methods Three consecutive cases of CS d-AVF with ipsilateral or bilateral IPS occlusion were involved in this study. On TVE, the orifice of the IPS was investigated with IVUS placed in the jugular vein or jugular bulb.

Results This technique has been successfully adapted in all three cases. In two of these cases, IPS was well visualized with the help of IVUS, and TVE was successfully performed. Conclusion To our knowledge, this is the first report to mention the usefulness of IVUS for detecting angiographically occult IPS.
\end{abstract}

Keywords Cavernous sinus dural arteriovenous fistula . Inferior petrosal sinus · Intravascular ultrasonography · Transvenous embolization

Shigeru Yamauchi

s-yamauchi-bigdipper@trad.ocn.ne.jp

1 Department of Neurosurgery, Hokuto Social Medical Corporation Hokuto Hospital, 7-5 Inada, Obihiro, Hokkaido 080-0033, Japan

2 Department of Neurology, Hokuto Social Medical Corporation Hokuto Hospital, Obihiro, Hokkaido, Japan

3 Department of Neurosurgery, Osaka City University Graduate School of Medicine, Osaka, Japan

4 Department of Neurosurgery, Ishikiriseiki Hospital, Higashiosaka, Osaka, Japan

\section{Introduction}

Cavernous sinus (CS) dural arteriovenous fistula (d-AVF) is a condition in which the dural arteriovenous shunts form around the CS. This condition sometimes causes severe ocular symptoms and cerebral venous congestion. Although CS d-AVFs are usually treated with transvenous embolization (TVE) via the inferior petrosal sinus (IPS), IPSs are sometimes thrombosed and angiographically invisible. In such cases, the first obstacle to TVE is detecting the entry to the IPS, and this can prove difficult due to angiographic invisibility and anatomical variations. We describe herein the usefulness of intravascular ultrasonography (IVUS) for detecting an angiographically invisible caudal end of the IPS.

\section{Materials and methods}

Three consecutive cases of CS d-AVF with ipsilateral or bilateral IPS occlusion were involved in this study. In all cases, primary treatment was attempted by TVE via the occluded IPS, and attempts were made to detect the orifice of the IPS with IVUS (Eagle Eye Platinum ${ }^{\circledR}$; Volcano, San Diego, CA) (Fig. 1).

\section{TVE procedure protocol}

TVE procedures were performed under general or local anesthesia with femoral puncture. A 6-Fr guiding catheter was placed in the ipsilateral external or common carotid artery via a left transfemoral approach for control arteriography. Another 6-Fr guiding sheath was placed in the ipsilateral jugular vein (JV) via a right transfemoral approach. Initially, a microcatheter and 300-cm 0.014-in. microguidewire were navigated to the ipsilateral transverse sinus, then the microcatheter was 
Fig. 1 a A picture of the IVUS imaging system (Volcano S5 ${ }^{\mathrm{TM}}$ ) and $\mathbf{b}$ the IVUS catheter (Eagle Eye Platinum ${ }^{\circledR}$ ) used in our cases The catheter is monorail type compatible with a 0.014-in. guidewire

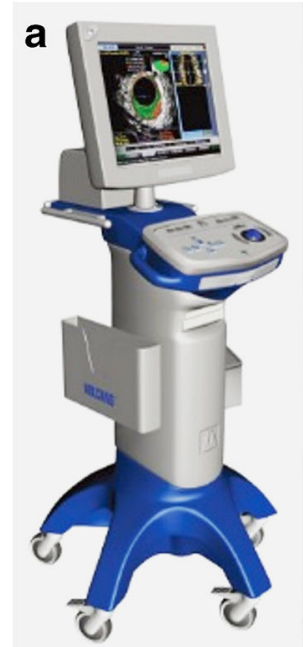

b

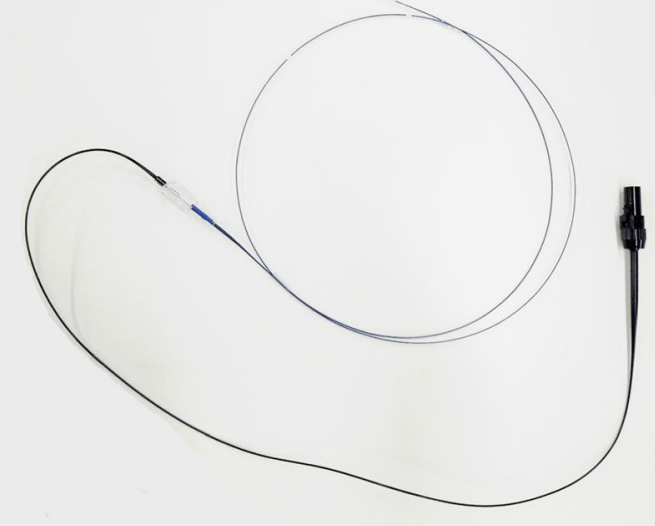

withdrawn with an exchange technique, and the IVUS catheter was advanced along with the microguidewire as distal as possible. The IVUS catheter was slowly pulled, and a gray-scale image and $\mathrm{ChromaFlo}^{\circledR}$ image were obtained. The rostral half of the JV and, if possible, the jugular bulb (JB) were investigated using this technique. Under this guidance, we tried to navigate the microcatheter to the IPS and performed TVE in the standard manner.

In this study, whether the IVUS could pass through the JB and whether the IVUS could detect the caudal end of the IPS were evaluated retrospectively.

\section{Results}

Among the three cases investigated, the tip of the IVUS catheter could not pass through the JB due to its tortuosity in case 1. In cases 2 and 3, the tip of the IVUS catheter was able to pass through the JB without difficulty. Although the JB and its surrounding structures were difficult to identify with IVUS in these two cases, the JV and surrounding tissue were clearly visualized with IVUS in all three cases.

In two of the three cases (cases 1 and 3), the caudal ends of the IPSs were clearly visualized with IVUS and ChromaFlo ${ }^{\circledR}$. These two cases both had low-set IPSs originating from the extracranial JV. In both cases, with the aid of IVUS, a microcatheter was easily navigated to the orifice of the IPS and finally to the CS.
In the remaining case (case 2), IVUS and ChromaFlo ${ }^{\circledR}$ failed to visualize the orifice of the IPS (Table 1). No procedure-related complications were encountered in any of the three cases.

\section{Case presentation}

\section{Case 1}

The patient was a 73-year-old woman who developed pulsatile tinnitus and diplopia 3 months before admission. Two months later, although tinnitus had disappeared, she had developed pulsatile headache. Diagnostic angiography revealed bilateral CS d-AVF, supplied by the dural branches of the bilateral external and internal carotid arteries. Shunt blood flow mainly drained through bilateral superficial middle cerebral veins (SMCVs) in a retrograde pattern. Bilateral IPSs and superior orbital veins (SOVs) were not able to be visualized angiographically (Fig. 2).

TVE through the left IPS was performed under general anesthesia in accordance with the protocol described above. In this case, the tip of the IVUS catheter could not pass through the JB due to its tortuosity. Almost the entire rostral half of the JV was investigated with IVUS, and the angiographically invisible IPS was visualized, identifying a lowset caudal end of the IPS. ChromaFlo ${ }^{\circledR}$ image could also detect the angiographically undetectable blood flow in the IPS (Fig. 3). Under this guidance, we were able to easily navigate

Table 1 Summary of the three cases

\begin{tabular}{|c|c|c|c|c|c|c|}
\hline & $\begin{array}{l}\text { Visualization of JB } \\
\text { and surrounding structures }\end{array}$ & $\begin{array}{l}\text { Visualization of JV and } \\
\text { surrounding structures }\end{array}$ & $\begin{array}{l}\text { Visualization of IPS } \\
\text { with gray scale }\end{array}$ & $\begin{array}{l}\text { Visualization of IPS } \\
\text { with ChromaFlo }{ }^{\circledR}\end{array}$ & Pushability through JB & Origin of IPS \\
\hline Case 1 & NA & Comprehensible & Enable & Enable & Unable & Extracranial \\
\hline Case 2 & Incomprehensible & Comprehensible & Unable & Unable & Enable & Undetected \\
\hline Case 3 & Incomprehensible & Comprehensible & Enable & Enable & Enable & Extracranial \\
\hline
\end{tabular}

$N A$ not available 
Fig. 2 Diagnostic angiography in case 1. Bilateral (a right, b left) common carotid angiography demonstrates CS d-AVF involving bilateral CSs. Shunt blood flow mainly drains to bilateral SMCVs in a retrograde pattern. Bilateral IPSs are not angiographically visualized

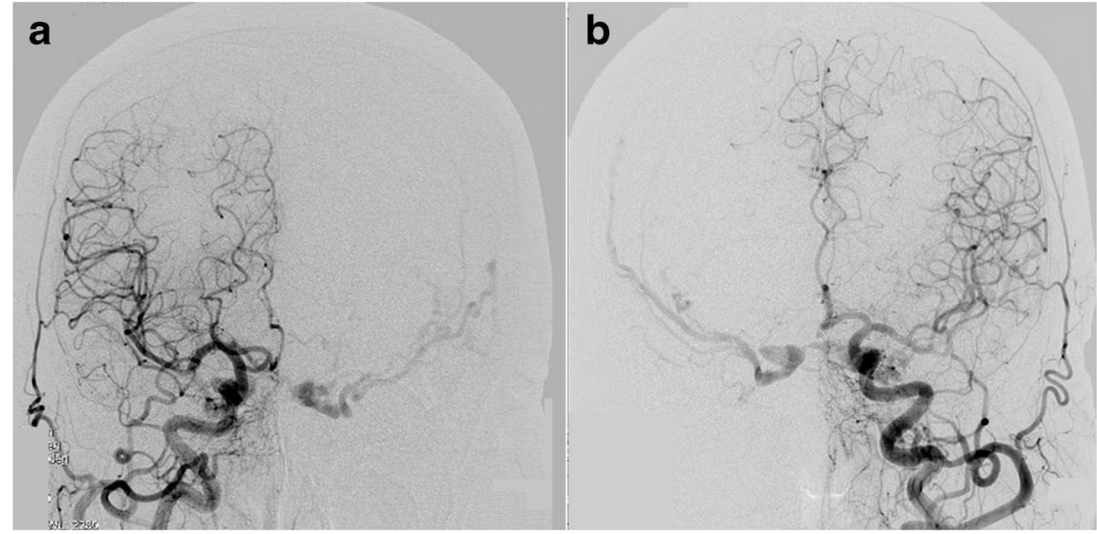

the microcatheter to the IPS and finally to the CS. Initially, we aimed to occlude the shunt point selectively, but this proved to be difficult. We therefore performed sinus packing, and the shunt was then completely obliterated.

\section{Case 2}

The patient was an 82-year-old woman. She developed diplopia and chemosis of both eyes 1 month before admission. Diagnostic angiography revealed CS d-AVF involving the CS bilaterally, supplied by the dural branches of the bilateral external and partially internal carotid arteries. Shunt blood flow mainly drained into bilateral SOVs in a retrograde pattern, with partial drainage through the retrograde leptomeningeal venous drainage to the posterior fossa. Bilateral IPSs were not able to be visualized angiographically (Fig. 4).

TVE through the right IPS was tried under local anesthesia in accordance with the protocol described. In this case, the tip of the IVUS catheter could pass through the JB without difficulty. Although the sigmoid sinus and JB were visualized with IVUS, the results were difficult to comprehend due to the surrounding bone structure and its tortuosity. On the other hand, the JV and surrounding tissue were clearly visualized with gray-scale and $\mathrm{ChromaFlo}^{\circledR}$ images, and the image was easily comprehended. In this case, we could not detect the entry of the IPS with IVUS. We subsequently searched for the orifice of the IPS in the usual manner for $1 \mathrm{~h}$, but failed and abandoned TVE. Palliative transarterial embolization (TAE) was performed using poly vinyl alcohol (PVA) particles. After the procedure, symptoms improved.

\section{Case 3}

The patient was a 73-year-old woman who had developed gait disturbance 2 weeks before admission. Diagnostic angiography revealed CS d-AVF involving the left cavernous sinus and
Fig. 3 IVUS images in case 1. Left $(\mathbf{a}, \mathbf{d})$ gray-scale image. Middle (b, e) ChromaFlo ${ }^{\circledR}$ image. Right (c, f) gray-scale image after wire insertion. Asterisks $(*)$ indicate IPS, and white arrows indicate the guidewire inserted into the IPS. The upper three images $(\mathbf{a}, \mathbf{b}, \mathbf{c})$ represent slices slightly distal to the orifice of the IPS, with the IPS is running parallel to the JV. The lower three images $(\mathbf{d}, \mathbf{e}, \mathbf{f})$ show slices at the orifice of the IPS

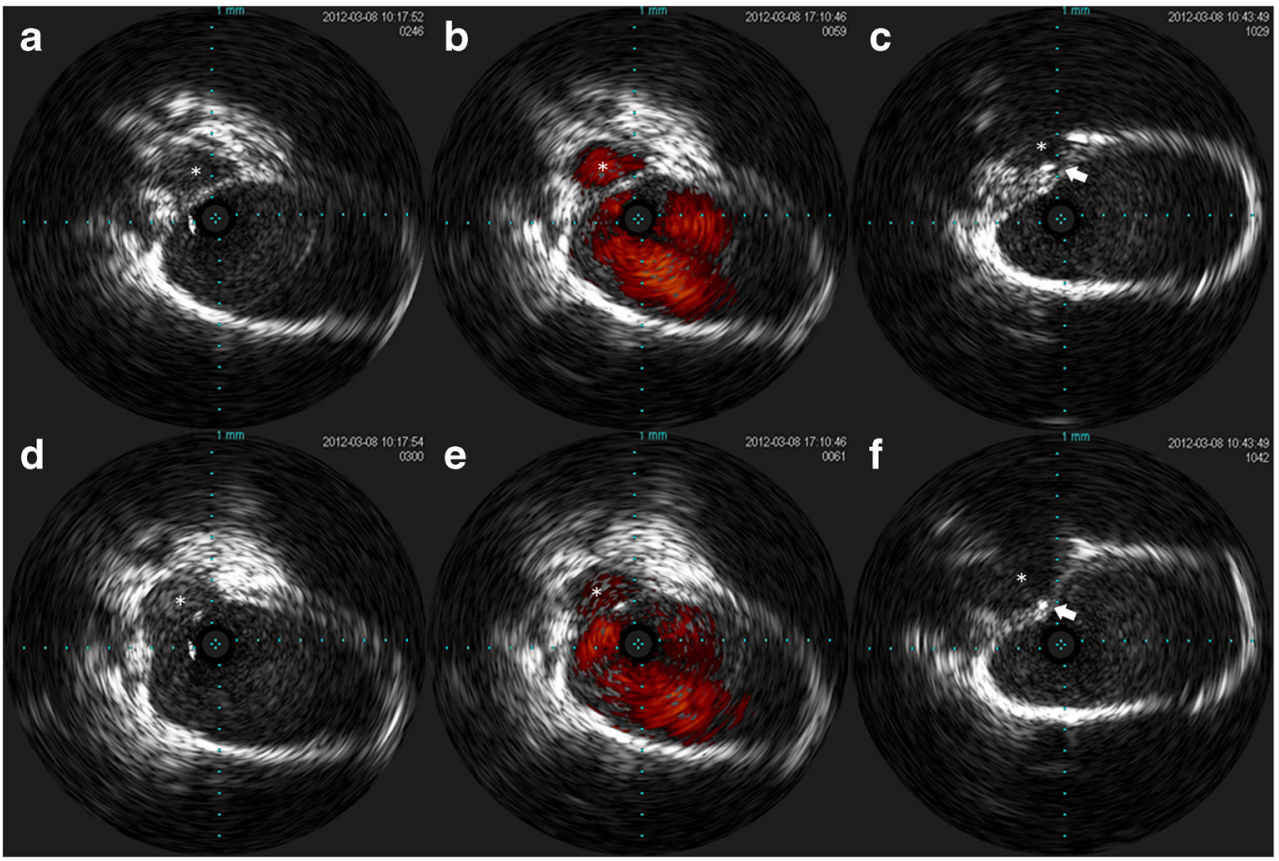


Fig. 4 Diagnostic angiography in case 2. Bilateral (a right, b left) common carotid angiography demonstrates CS d-AVF involving bilateral CSs. Shunt blood flow mainly drains through bilateral SOVs in a retrograde pattern and partially draining into the leptomeningeal venous drainage of the posterior fossa. Bilateral IPSs are not seen angiographically

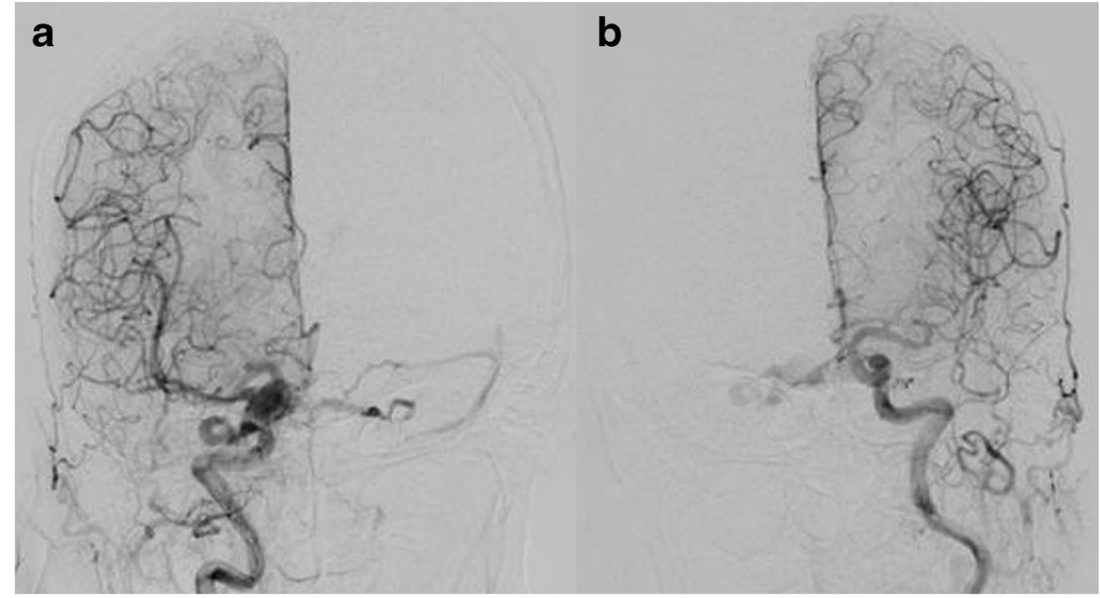

supplied by the dural branches of the left external and internal carotid arteries. Shunt blood flow drained into the left petrosal vein via the left superior petrosal sinus in a retrograde manner. Ipsilateral IPS was not angiographically visualized (Fig. 5).

TVE through the left IPS was performed under general anesthesia using the described protocol. In this case, the tip of the IVUS catheter was also able to easily pass through the JB. Although the sigmoid sinus and JB were visualized on IVUS, comprehension of the results was difficult, as in case 2. The JV and surrounding tissue were again clearly visualized with gray-scale and ChromaFlo ${ }^{\circledR}$ images. In this case, the low-set entry of the IPS was clearly identified in gray scale and the angiographically undetectable blood flow in the IPS could be detected on ChromaFlo ${ }^{\circledR}$ images (Fig. 6). Under this guidance, we were able to easily navigate the microcatheter to the IPS and finally to the CS. Shunt blood flow was successfully obliterated.

\section{Discussion}

To perform TVE via a thrombosed IPS, initially, the orifice of the angiographically invisible IPS must be located. Operators generally rely only on anatomical knowledge and palpation by hand to identify the IPS, but this is sometimes difficult due to its invisibility and anatomical variations.

Several reports have described anatomical variations to the caudal end of the IPS [1, 2]. Mitsuhashi et al. [2] recently investigated this variation with rotation digital-subtraction venography and proposed a new classification. According to their study, $3.6 \%$ of cases showed no direct connection between the IPS and the JV or JB (type E), and $16.9 \%$ showed no IPS (type F) [2]. With these types, detecting the entry point to the occluded IPS with standard techniques might be impossible or very difficult. If we could determine whether a direct connection between JV and IPS existed, we would be able to save time and avoid unnecessary irradiation. We therefore considered new methods for detecting the invisible origin of the IPS.

At the end of the 1980s, IVUS was introduced as a new modality to visualize vessel walls and their characteristics $[3,4]$. Since then, this modality has predominantly been used for atherosclerotic stenosis or arterial dissection in interventional radiology and cardiology [5-10].
Fig. 5 Diagnostic left common carotid angiography in case 3 (a $A P$ view, b lateral view). CS d-AVF involves the left cavernous sinus and is supplied by the dural branches of the left external and internal carotid arteries. Shunt blood flow drains into the left petrosal vein via the left superior petrosal sinus in a retrograde pattern. The ipsilateral IPS is not angiographically visualized
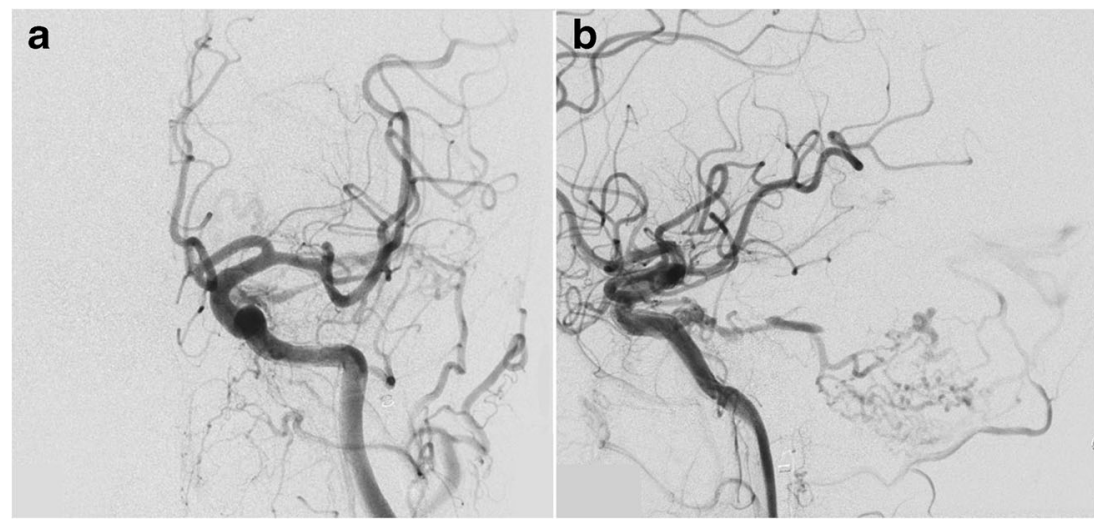
Fig. 6 IVUS image in case 3. Upper images $(\mathbf{a}, \mathbf{b}, \mathbf{c})$ gray-scale images. Lower images $(\mathbf{d}, \mathbf{e}, \mathbf{f})$ ChromaFlo ${ }^{\circledR}$ images. Right (a, d) a slice of the JB, in which the vessel wall of the JB is visualized, but the surrounding tissue is not identifiable. Middle (b, e) a slice slightly distal to the orifice of the IPS, in which the IPS running parallel to the JV is clearly demonstrated in both images. Left (c, $\mathbf{f})$ just the slice of the orifice of the IPS. Asterisks $(*)$ indicate IPS

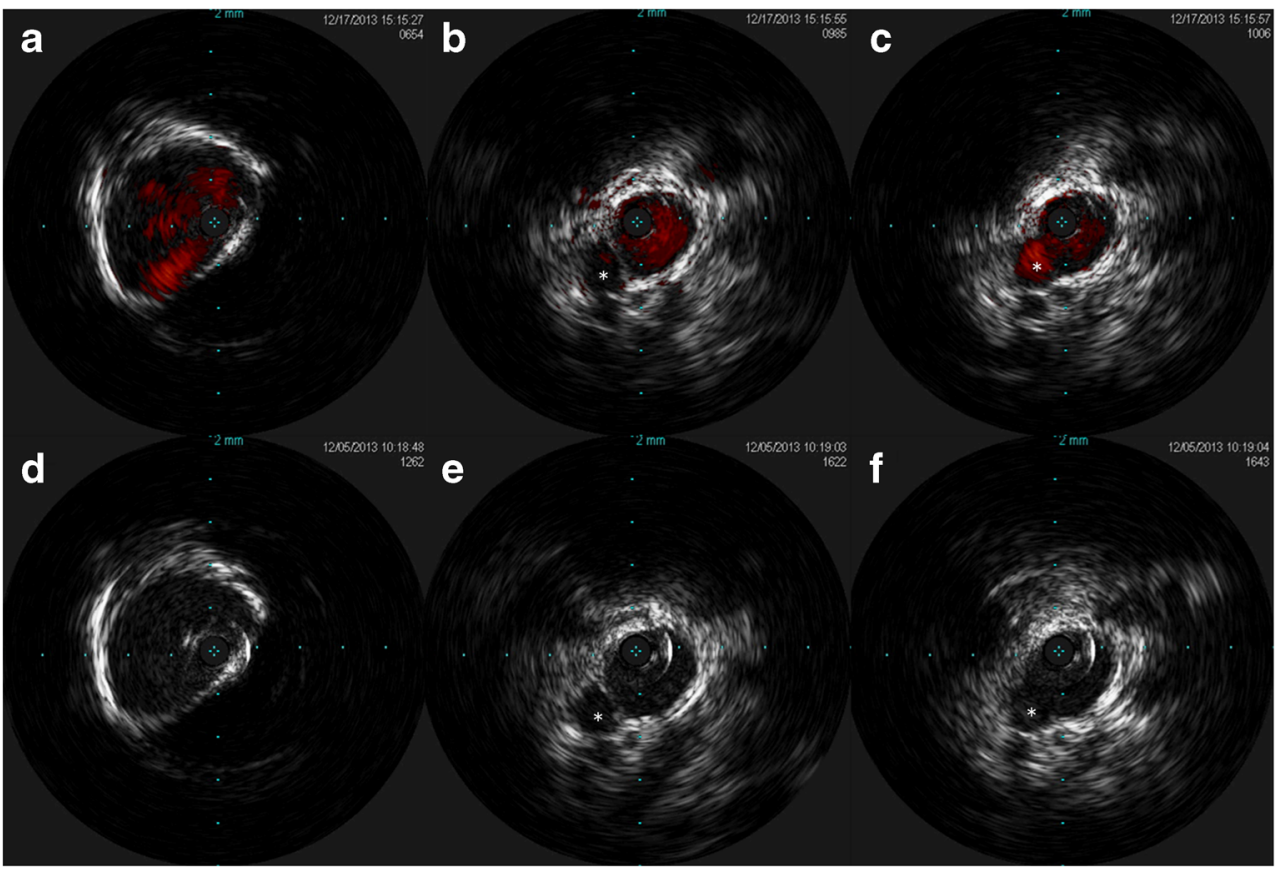

Some reports have described other usages of IVUS. Nishio et al. reported the utility of IVUS for monitoring herniated coils in the embolization of direct carotid cavernous fistula [11]. Yoshioka et al. also reported TAE of direct vertebral arteriovenous fistula with the aid of IVUS [12]. In the field of coronary intervention, some technical reports have described chronic total occlusion in which target vessels were visualized with the IVUS catheter placed in other vessels [13, 14].

IVUS can visualize not only the vessel wall but also the surrounding structures. This property of IVUS has been applied to the techniques described by other investigators. Our technique also followed this concept.

In this study, IVUS was able to visualize the JV and surrounding tissue clearly in all three cases and could detect the extracranial origin of the IPS in two cases. In those two cases, IVUS could also detect the angiographically invisible blood flow in the caudal end of the IPS with ChromaFlo ${ }^{\circledR}$. IVUS might represent a useful modality for detecting an extracranial origin of the IPS.

On the other hand, the JB may sometimes be difficult to pass through with IVUS catheter for its tortuosity. In one of our three cases, we could not navigate the tip of IVUS catheter to the sigmoid sinus. In such a case, a stiffer guiding catheter or direct puncture of JV might be needed to pass through the tortuous JB. In the remaining two cases, navigation of the tip of the IVUS catheter to the sigmoid sinus was easily carried out even under local anesthesia. Although the JB was investigated with IVUS in those two cases, IVUS images were difficult to interpret in this portion. The JB is anatomically tortuous and covered with bone. When we pulled back the IVUS catheter, as the tip was caught by the tortuosity of the JB, moving the tip in a smooth manner proved difficult. The surrounding bone interfered with clear vision with its acoustic shadow. Moreover, as the JB has multiple branches, distinguishing the IPS from other branches such as the marginal sinus, posterior condylar vein, and petroclival vein might be difficult with IVUS. From these findings, IVUS might not be particularly useful for detecting an intracranial origin of the IPS. At present, this might represent a major limitation of our technique. To overcome this limitation, other methods such as optical coherence tomography or high-frequency IVUS might be applicable.

Our technique thus remains incomplete in terms of reliably detecting IPS in all cases. However, according to the report by Mitsuhashi et al., the IPS originates from the extracranial JV in about $70 \%$ of cases, so if an extracranial origin of the IPS could be reliably detected with this technique, at least $70 \%$ of cases might benefit [2]. IVUS would be one useful option for such cases.

\section{Conclusion}

This is the first report to describe the use of IVUS for detecting an occluded IPS. Although IVUS could detect a low-set IPS, detecting an intracranial origin of the IPS is difficult with this technique. As this report involves only a small number of cases, and represents only a preliminary experience, accumulation of more cases will clarify whether this technique is useful. 
Ethical standards and patient consent We declare that this study has been approved by the Hokuto Ethics Committee and has therefore been performed in accordance with the ethical standards laid down in the 1964 Declaration of Helsinki and its later amendments. We declare that all patients gave informed consent prior to inclusion in this study.

Conflict of interest We declare that we have no conflict of interest.

Open Access This article is distributed under the terms of the Creative Commons Attribution 4.0 International License (http://creativecommons.org/ licenses/by/4.0/), which permits unrestricted use, distribution, and reproduction in any medium, provided you give appropriate credit to the original author(s) and the source, provide a link to the Creative Commons license, and indicate if changes were made.

\section{References}

1. Miller DL, Doppman JL, Chang R (1993) Anatomy of the junction of the inferior petrosal sinus and the internal jugular vein. AJNR Am J Neuroradiol 14(5):1075-1083

2. Mitsuhashi Y, Nishio A, Kawahara S, Ichinose T, Yamauchi S, Naruse H, Matsuoka Y, Ohata K, Hara M (2007) Morphologic evaluation of the caudal end of the inferior petrosal sinus using 3D rotational venography. AJNR Am J Neuroradiol 28(6):11791184. doi:10.3174/ajnr.A0489

3. Neville RF, Bartorelli AL, Sidawy AN, Almagor Y, Potkin B, Leon MB (1989) An in vivo feasibility study of intravascular ultrasound imaging. Am J Surg 158(2):142-145

4. Gussenhoven EJ, Essed CE, Lancee CT, Mastik F, Frietman P, van Egmond FC, Reiber J, Bosch H, van Urk H, Roelandt J et al (1989) Arterial wall characteristics determined by intravascular ultrasound imaging: an in vitro study. J Am Coll Cardiol 14(4):947-952

5. Iwabuchi M, Haruta S, Taguchi A, Ichikawa Y, Genda T, Katai S, Imaoka T, Shimizu Y, Owa M (1997) Intravascular ultrasound findings after successful primary angioplasty for acute myocardial infarction: predictors of abrupt occlusion. J Am Coll Cardiol 30(6): $1437-1444$
6. Abizaid A, Pichard AD, Mintz GS, Abizaid AS, Klutstein MW, Satler LF, Mehran R, Leiboff B, Kent KM, Leon MB (1999) Acute and long-term results of an intravascular ultrasound-guided percutaneous transluminal coronary angioplasty/provisional stent implantation strategy. Am J Cardiol 84(11):1298-1303

7. Buckley CJ, Arko FR, Lee S, Mettauer M, Little D, Atkins M, Manning LG, Patterson DE (2002) Intravascular ultrasound scanning improves long-term patency of iliac lesions treated with balloon angioplasty and primary stenting. J Vasc Surg 35(2):316-323

8. Ohshima K, Ikeda S, Watanabe K, Yamane K, Izumi N, Ishibashi K, Ohshima K, Hamada M (2009) Relationship between plaque composition and no-reflow phenomenon following primary angioplasty in patients with ST-segment elevation myocardial infarction - analysis with virtual histology intravascular ultrasound. J Cardiol 54(2):205-213. doi:10.1016/j.jjcc.2009.05.009

9. Maehara A, Mintz GS, Castagna MT, Pichard AD, Satler LF, Waksman R, Suddath WO, Kent KM, Weissman NJ (2002) Intravascular ultrasound assessment of spontaneous coronary artery dissection. Am J Cardiol 89(4):466-468

10. Koschyk DH, Meinertz T, Hofmann T, Kodolitsch YV, Dieckmann C, Wolf W, Knap M, Kiraly Z, Steffen W, Beythien C, Lund G, Rehders T, Nienaber CA (2003) Value of intravascular ultrasound for endovascular stent-graft placement in aortic dissection and aneurysm. J Card Surg 18(5):471-477

11. Nishio A, Kawakami T, Mitsuhashi Y, Hayasaki K, Kiyama M, Tada Y, Ohata K (2009) Usefulness of intravascular ultrasonography monitoring of coil embolization for traumatic direct carotidcavernous fistula-case report. Neurol Med Chir 49(12):604-607

12. Yoshioka T, Kitagawa N, Morikawa M, Hayashi K, Izumo T, Nakamoto M, Kaminogo M, Nagata I (2004) Successful transarterial occlusion of a vertebral arteriovenous fistula with aid of intra vascular ultra sound (IVUS) and balloon catheter. No Shinkei Geka Neurol Surg 32(6):597-602

13. Park Y, Park HS, Jang GL, Lee DY, Lee H, Lee JH, Kang HJ, Yang DH, Cho Y, Chae SC, Jun JE, Park WH (2011) Intravascular ultrasound guided recanalization of stumpless chronic total occlusion. Int J Cardiol 148(2):174-178. doi:10.1016/j.ijcard.2009.10.052

14. Takahashi Y, Okazaki H, Mizuno K (2013) Transvenous IVUSguided percutaneous coronary intervention for chronic total occlusion: a novel strategy. J Invasive Cardiol 25(7):E143-E146 